\title{
A Swarm Aggregation Algorithm based on Local Interaction with Actuator Saturations and Integrated Obstacle Avoidance
}

\author{
Antonio Leccese ${ }^{1}$, Andrea Gasparri ${ }^{1}$, Attilio Priolo ${ }^{1}$, Giuseppe Oriolo ${ }^{2}$ and Giovanni Ulivi ${ }^{1}$
}

\begin{abstract}
In this paper, a novel decentralized swarm aggregation algorithm for multi-robot systems with an integrated obstacle avoidance is proposed. In this framework, the interaction among robots is limited to their visibility neighborhood, i.e., robots that are within the visibility range of each other. Furthermore, to better comply with the hardware/software limitations of mobile robotic platforms, robots actuators are assumed to be saturated. A theoretical characterization of the main properties of the proposed swarm aggregation algorithm is provided. Simulations have been carried out to validate the theoretical results and experiments have been performed with a team of low-cost mobile robots to demonstrate the effectiveness of the proposed approach in real scenario.
\end{abstract}

\section{INTRODUCTION}

Multi-robot systems have been a very attractive research area in the last two decades. The attention given by the robotics community to this research field is motivated by the wide range of applications which can be carried out by a team of robots, such as environmental exploration [1], search and rescue operations [2], coverage tasks [3], agricultural foraging [4] or sweeping operations [5].

Several important research problems arise in multi-robot systems, ranging from localization [6], [7], task sequencing and mission-control [8], [9] to decentralized coordination [10], [11], [12], [13]. In this work, we consider the decentralized coordination problem for swarm robotics. Swarm robotics can be defined as the study of how a group of relatively simple, low-cost robots can be constructed to collectively accomplish tasks that are beyond the capabilities of a single one while providing a higher robustness and flexibility. The reader is referred to [14], [15] for a complete overview on swarm robotics.

Different swarm aggregation algorithms can be found in the literature [16], [17], [18], [19]. Some of these algorithms considers also actuator saturations [20], [21], [22].

In this paper, we present a novel decentralized swarm aggregation algorithm for team of robots with limited onehop interactions and actuators saturation. This work, compared to our previous contributions [23], [24], presents two major novelties: i) the actuator saturation can be asymmetric

\footnotetext{
${ }^{1}$ A. Leccese, A. Gasparri, A. Priolo and G. Ulivi are with the Department of Engineering, University of "Roma Tre", Via della Vasca Navale, 79. Roma, 00146, Italy. Emails: \{leccese, gasparri, priolo, ulivi\}@dia.uniroma3. it

${ }^{2}$ G. Oriolo is with the "Dipartimento di Ingegneria Informatica, Automatica e Gestionale", University of Rome "La Sapienza", via Ariosto 25, 00185 Roma, Italy. Email: oriolo@dis . uniroma1. it

This work was partially supported by the Italian grant FIRB "Futuro in Ricerca", project NECTAR "Networked Collaborative Team of Autonomous Robots", code RBFR08QWUV, funded by the Italian Ministry of Research and Education (MIUR).
}

with respect to the forward and backward velocity to better comply with the hardware/software specifications, and ii) the obstacle avoidance algorithm can be easily integrated within the local control law of each robot ensuring a safer navigation in an environment filled with obstacles.

\section{PRELIMINARIES}

Let us consider a swarm composed of $n$ robot whose interaction can be modeled by means of an undirected time-varying proximity graph $\mathcal{G}(t)=\{V, E(t)\}$ where $V=\{1, \ldots, n\}$ are the vertices (robots) and $E(t)=\left\{\epsilon_{i j}(t)\right\}$ the set of edges representing the interaction between pairs of robots. An edge $\epsilon_{i j}(t)$ exists between a pair of robots $i$ and $j$ if and only if they are within the range of visibility of each other, i.e., $\epsilon_{i j}(t)=1 \Leftrightarrow\left\|x_{i}(t)-x_{j}(t)\right\| \leq r$, otherwise $\epsilon_{i j}(t)=0$, where $x_{i}(t) \in \mathbb{R}^{d}$ is the location of the $i$-th robot, $\|\cdot\|$ is the Euclidean norm and $r$ is the range of visibility. Let us denote with $\mathcal{A}(\mathcal{G}(t))$ the adjacency matrix of $\mathcal{G}(t)$ whose entries $a_{i j}(t)=1 \Leftrightarrow \epsilon_{i j}(t)=1$, otherwise $a_{i j}(t)=0$. Furthermore, let us denote the set of neighboring robots for the $i$-th robot with $\mathcal{N}_{i}(t)=\left\{j \in V \backslash\{i\}: \epsilon_{i j}(t)=1\right\}$. In addition, let us denote with $\mathcal{D}(\mathcal{G}(t))=\operatorname{diag}\left(D_{1}(t), \ldots, D_{n}(t)\right)$ the degree matrix of $\mathcal{G}(t)$, where $D_{i}(t)=\left|\mathcal{N}_{i}(t)\right|$ is the degree of the $i$-th robot. Finally, let us denote with $\mathcal{L}(\mathcal{G}(t))=$ $D(\mathcal{G}(t))-A(\mathcal{G}(t))$ the $n \times n$ Laplacian matrix of $\mathcal{G}(t)$. For the sake of readability the notation $\mathcal{L}(t), \mathcal{A}(t), \mathcal{D}(t)$ will be used in the rest of the paper.

Let us now review some important properties of the Laplacian matrix $\mathcal{L}$. First, let us recall that $\mathcal{L}$ is a weakly diagonal dominant symmetric matrix for which both the row sum and the column sum are equal to zero. This implies that there is always at least a zero structural eigenvalue whose corresponding eigenvector is $\mathbf{1}$, the $n$-vector with all unit components. Thus $\mathcal{L}(\mathcal{G}) \mathbf{1}=\mathbf{0}$ and $\mathbf{1}^{T} \mathcal{L}(\mathcal{G})=\mathbf{0}^{T}$ for any $\mathcal{G}$. Furthermore, the number of zero eigenvalues corresponds to the number of connected components of $\mathcal{G}$, that is $\operatorname{Rank}(\mathcal{L}(\mathcal{G}))=n-c$, with $c$ the number of connected components. In addition, according to the Gershgorin Circle Theorem, all the eigenvalues of the Laplacian are real and positive, and in particular belong to $\left[0,2 D_{\max }(\mathcal{G})\right]$, where $D_{\max }(\mathcal{G})=\max _{i \in V}\left\{D_{i}(\mathcal{G})\right\}$ is the maximum degree among the nodes in the graph. Among the others, the second smallest eigenvalue $\lambda_{2}(\mathcal{L})$, called algebraic connectivity, plays a key role as it provides information about the connectedness of the graph. For the sake of readability, the timedependency will be omitted in the following if not strictly required. 


\section{Aggregation Control LaW}

Let us consider a swarm of $n$ mobile robots for which, as detailed in Section II, the interaction is described by means of an undirected time-varying proximity graph $\mathcal{G}(t)=\{V, E(t)\}$. In particular, for each mobile robot $i$ consider the following dynamics:

$$
\dot{x}_{i}=\frac{\sum_{j \in \mathcal{N}_{i}(t)} \gamma_{i j} g\left(x_{i}-x_{j}\right)}{\sum_{j \in \mathcal{N}_{i}(t)} \gamma_{i j}}
$$

where $\gamma_{i j}$ is the weighting factor between each pair of neighboring robots $i$ and $j$ defined as:

$$
\gamma_{i j}=\frac{1}{\left\|x_{i}-x_{j}\right\|^{\alpha}}, \quad \text { with } \alpha \geq 1,
$$

and $g(\cdot)$ is the interaction function defined as:

$$
g(y)=\frac{y}{\|y\|}\left[\left(g_{r}(\|y\|)-g_{a}(\|y\|)\right)\right], \quad y \in \mathbb{R}^{d}
$$

with $g_{a}(\cdot): \mathbb{R} \rightarrow \mathbb{R}$ and $g_{r}(\cdot): \mathbb{R} \rightarrow \mathbb{R}$ the attraction and repulsion functions, respectively. Note that, by construction the interaction function is odd, that is $g(y)=-g(-y)$. In particular, the attraction and repulsion functions are defined as follows:

$$
\begin{aligned}
& g_{a}(\|y\|)=a(1-\Phi(\|y\|)), \\
& g_{r}(\|y\|)=b \Phi(\|y\|),
\end{aligned}
$$

where $a, b>0$ are constant values and $\Phi(\cdot): \mathbb{R} \rightarrow \mathbb{R}$ a suitable generalized function. Note that, the term $g_{r}(\|y\|)-g_{a}(\|y\|)$ by construction takes values within the range $[-a, b]$.

The following assumptions are made on the attractive and the repulsive functions and on the generalized function $\Phi(\cdot)$, respectively.

Assumption 1: There exists a unique distance $\delta$ at which the attractive and repulsive functions balance, i.e., $g_{a}(\delta)=g_{r}(\delta)$. Furthermore, it is $g_{a}(\|y\|) \geq g_{r}(\|y\|)$ for $\|y\| \geq \delta$ and $g_{a}(\|y\|)<g_{r}(\|y\|)$ for $\|y\|<\delta$.

Assumption 2: A generalized function $\Phi(\cdot)$ must satisfy the following properties:

- monotonic function

- $\lim _{\|y\| \rightarrow 0} \Phi(\|y\|)=1$

- $\lim _{\|y\| \rightarrow \infty} \Phi(\|y\|)=0$

Example 1: As an example of generalized function $\Phi(\cdot)$, let us consider:

$$
\begin{aligned}
& \text { - } \exp \left(-\frac{\|y\|^{\beta}}{c}\right) \text { with } \beta \geq 1 \text { and } c>0, \\
& \text { - } \operatorname{sech}\left(-\|y\|^{\beta}\right) \text { with } \beta \geq 1 \text {. }
\end{aligned}
$$

As pointed out in the introduction, compared to the approaches for swarm aggregation we previously proposed in [23], [24] the control law given in eq. (1) has two major advantages:
1) It allows the input saturation to be asymmetric with respect to the forward and backward velocity to better comply with the hardware/software specifications.

2) It allows an easier and yet more natural integration of the obstacle avoidance.

Let us now focus on the obstacle avoidance integration. In [24], the authors introduce an effective way to integrate the obstacle avoidance. An obstacle is represented as a set of virtual robots created on the boundary of the closest obstacle by projection, i.e. they are located on the boundary of the obstacle at the minimum distance from the detecting robot. Furthermore, the cardinality of this set of virtual robots has to be at least equal to the current neighborhood of the considered robot. Indeed, this would prevent any collision because the repulsion originated by the virtual robots will certainly counteract any possible influence by the actual neighbors. Note that, to avoid an undesired attraction to the obstacle, the activation threshold for the obstacle avoidance algorithm must be set to the distance for which the repulsive and attractive actions balance.

In this work, instead, by using the control law given in eq. (1) only one virtual robot has to be projected on the closest obstacle. This can be explained by the fact that the control law proposed in this work relies on the presence of weighting factors $\gamma_{i k}$. To better understand this concept let us denote with $x_{k}$ the location of a virtual robot representing an obstacle detected by a given robot $i$. It is worthy to recall that, also in this case, the virtual robot is projected on the closest obstacle only if the distance from the actual robot to the obstacle itself is shorter than equilibrium distance of (1), i.e., the distance for which the repulsive and attractive actions balance. Then, if the robot $i$ moves close to the virtual robot $x_{k}$ the term $\gamma_{i k}=\frac{1}{\left\|x_{i}-x_{k}\right\|^{\alpha}}$ increases, therefore the control contribution due to the interaction with the virtual robot becomes more relevant and this allows to prevent a collision with the virtual robot $k$ associated to a certain obstacle regardless of the cardinality of the current true neighborhood of robot $i$. Obviously, for the sake of the input saturation, this procedure requires a normalization term $\gamma_{i}$ for each robot $i$ to generate a feasible control input, that is:

$$
\gamma_{i}=\sum_{j \in \mathcal{N}_{i}(t)} \gamma_{i j}=\sum_{j \in \mathcal{N}_{i}(t)} \frac{1}{\left\|x_{i}-x_{j}\right\|^{\alpha}}>0 .
$$

Note that, in this work obstacles are assumed to be convex in order to ensure the repulsive action to be continuous. In the case of not convex obstacles, it is always possible to resort to well known convex decomposition techniques, see [25, Chapter 7], so that this assumption can be satisfied.

In the following, the main properties of the proposed swarm aggregation algorithm will be proven. In that view, let us now introduce some useful tools for the analysis. In particular, let us denote with $\bar{x}(t)=\frac{1}{n} \sum_{i=1}^{n} x_{i}(t)$ the barycenter of the swarm and with $e_{i}(t)=x_{i}(t)-\bar{x}(t)$ the vector distance of robot $i$ from the barycenter $\bar{x}(t)$. Also, let us denote with $e(t)=\left[e_{1}(t) \ldots e_{n}(t)\right]^{T}$ the collection of all the distances 
from the barycenter and with $\chi(t)=\left[x_{1}(t) \ldots x_{n}(t)\right]^{T}$ the collection of all the agents locations.

The following assumptions will be considered in the rest of the paper for the sake of the analysis.

Assumption 3: The graph $\mathcal{G}(t)$ remains connected all the time. Furthermore, it is $\lambda_{2}(\hat{\mathcal{L}}(t)) \geq \lambda_{2, \min }(\hat{\mathcal{L}}(t))$ with $\hat{\mathcal{L}}$ the error Laplacian matrix whose elements definition is given in eq. (12).

Assumption 4: There exist functions $J_{a}(\|y\|): \mathbb{R}^{+} \rightarrow \mathbb{R}^{+}$ and $J_{r}(\|y\|): \mathbb{R}^{+} \rightarrow \mathbb{R}^{+}$such that:

$$
\begin{aligned}
& \nabla_{y} J_{a}(\|y\|)=\left(\frac{1}{\|y\|}\right)^{\alpha} \frac{y}{\|y\|} g_{a}(y), \\
& \nabla_{y} J_{r}(\|y\|)=\left(\frac{1}{\|y\|}\right)^{\alpha} \frac{y}{\|y\|} g_{r}(y) .
\end{aligned}
$$

\section{A. Steady State Analysis}

In the following we prove that the multi-robot system always reaches a steady state equilibrium for any given initial condition.

Theorem 1: Consider a swarm of robots whose dynamics is described by eq. (1). Then the swarm converges to an equilibrium state for any initial condition.

Proof: Define $J(\|y\|)=J_{a}(\|y\|)-J_{r}(\|y\|)$, with $J_{a}(\cdot)$ and $J_{r}(\cdot)$ as in Assumption 4. Consider the following (generalized) common Lyapunov candidate $V(t)=\frac{1}{2} \sum_{(i, j) \in E(t)} J\left(\left\|x_{i}(t)-x_{j}(t)\right\|\right)$ whose time derivative is:

$$
\begin{aligned}
\dot{V}(t) & =\sum_{i=1}^{n}\left(\nabla_{x_{i}} V(t)\right)^{T} \dot{x}_{i}(t) \\
& =-\sum_{i=1}^{n} \sum_{j \in \mathcal{N}_{i}(t)} \gamma_{i j}\left\|\dot{x}_{i}\right\|^{2} \leq 0 .
\end{aligned}
$$

being $\nabla_{x_{i}} V(t)=-\sum_{j \in \mathcal{N}_{i}(t)} \gamma_{i j} \dot{x}_{i}(t)$. Hence, using LaSalle's Invariance Principle, it follows that as $t \rightarrow \infty$ the state $\chi(t)$ converges towards the largest invariant subset of the set where $\dot{V}(t)=0$, that is:

$$
\Omega_{e}=\{\chi: \dot{\chi}(t)=0\},
$$

which proves the thesis as $\Omega_{e}$ is made of equilibrium points.

Example 2: Consider the following generalized function $\Phi(\|y\|)=\exp \left(-\frac{\|y\|^{4}}{c}\right)$. Then, the Assumption 4 is satisfied and the following (generalized) Lyapunov candidate can be used in Theorem 1:

$$
\begin{aligned}
V(t) & =\sum_{(i, j) \in E(t)}\left[-\frac{a}{2\left\|x_{i}-x_{j}\right\|^{4}}\right. \\
& \left.+(a-b)\left(-\frac{e^{-\frac{\left\|x_{i}-x_{j}\right\|^{4}}{c}}}{2\left\|x_{i}-x_{j}\right\|^{2}}-\frac{\sqrt{\pi} \operatorname{Erf}\left(\frac{\left\|x_{i}-x_{j}\right\|^{2}}{\sqrt{c}}\right)}{2 \sqrt{c}}\right)\right]
\end{aligned}
$$

with $\operatorname{Erf}(y)=\frac{2}{\sqrt{\pi}} \int_{0}^{y} e^{-t^{2}} d t$ the error function.

\section{B. Cohesiveness Analysis}

In the following we prove that the multi-robot system converges towards a bounded region whose size is a function of the parameters of the interaction function and of the network topology.

Theorem 2: Let us consider a swarm of robots whose dynamics is described by eq. (1). Then the swarm moves towards and remains within a bounded region:

$$
\mathcal{B}_{r}=\left\{\|\chi(t)-\bar{\chi}\| \leq \frac{\sqrt{n}}{\lambda_{2, \min }(\hat{\mathcal{L}})}\left(1+\frac{b}{a}\right)\right\}
$$

with $\bar{\chi}=\mathbf{1} \otimes \bar{x}$.

Proof: Let us take into account the following Lyapunov candidate function $V=\sum_{i} \frac{1}{2} e_{i}^{T} e_{i}$, whose time derivative is:

$$
\dot{V}=\sum_{i} e_{i}^{T} \dot{e}_{i}=\sum_{i} e_{i}^{T}\left(\dot{x}_{i}-\dot{\bar{x}}\right)=\underbrace{\sum_{i} e_{i}^{T} \dot{x}_{i}}_{V_{1}}-\underbrace{\sum_{i} e_{i}^{T} \dot{\bar{x}}}_{V_{2}} .
$$

We know from [24] that due to the limited interaction range and to the saturation effects, the barycenter is no longer stationary. Nevertheless, the dynamics of the barycenter does not affect the value of the time derivative of the Lyapunov function, namely:

$$
V_{2}=\sum_{i} e_{i}^{T} \dot{\bar{x}}=\left(\sum_{i} e_{i}^{T}\right)(\dot{\bar{x}})=\mathbf{0}^{T}(\dot{\bar{x}})=0 .
$$

Let us now consider the contribution $V_{i}$ given by the $i$-th robot to the term $V_{1}$ :

$$
\begin{aligned}
\dot{V}_{i}=-\frac{e_{i}^{T}}{\gamma_{i}} & {\left[\sum_{j \in \mathcal{N}_{i}(t)} \gamma_{i j} \frac{\left(x_{i}-x_{j}\right)}{\left\|x_{i}-x_{j}\right\|} g_{a}\left(\left\|x_{i}-x_{j}\right\|\right)\right.} \\
& \left.-\sum_{j \in \mathcal{N}_{i}(t)} \gamma_{i j} \frac{\left(x_{i}-x_{j}\right)}{\left\|x_{i}-x_{j}\right\|} g_{r}\left(\left\|x_{i}-x_{j}\right\|\right)\right]
\end{aligned}
$$

At this point, by substituting $g_{a}=a\left(1-\Phi\left(\left\|x_{i}-x_{j}\right\|\right)\right)$ and $g_{r}=b \Phi\left(\left\|x_{i}-x_{j}\right\|\right)$ in the previous equation it follows:

$$
\begin{aligned}
\dot{V}_{i}=- & \frac{e_{i}^{T}}{\gamma_{i}}\left[\sum_{j \in \mathcal{N}_{i}(t)} \gamma_{i j} \frac{\left(x_{i}-x_{j}\right) a\left(1-\Phi\left(\left\|x_{i}-x_{j}\right\|\right)\right)}{\left\|x_{i}-x_{j}\right\|}\right. \\
& \left.-\sum_{j \in \mathcal{N}_{i}(t)} \gamma_{i j} \frac{\left(x_{i}-x_{j}\right) b \Phi\left(\left\|x_{i}-x_{j}\right\|\right)}{\left\|x_{i}-x_{j}\right\|}\right] \\
\leq- & \frac{a}{\gamma_{i}} \sum_{j \in \mathcal{N}_{i}(t)} \gamma_{i j} \frac{e_{i}^{T}\left(x_{i}-x_{j}\right)}{\left\|x_{i}-x_{j}\right\|} \\
& +\frac{(a+b)}{\gamma_{i}} \sum_{j \in \mathcal{N}_{i}(t)} \gamma_{i j}\left\|e_{i}\right\| \Phi\left(\left\|x_{i}-x_{j}\right\|\right)
\end{aligned}
$$


Now, by considering that $\Phi\left(\left\|x_{i}-x_{j}\right\|\right) \leq 1$, the following holds:

$$
\dot{V}_{i} \leq-\frac{a}{\gamma_{i}} \sum_{j \in \mathcal{N}_{i}(t)} \gamma_{i j} \frac{e_{i}^{T}\left(x_{i}-x_{j}\right)}{\left\|x_{i}-x_{j}\right\|^{\hat{\alpha}}}+\frac{(a+b)}{\gamma_{i}}\left\|e_{i}\right\| \sum_{j \in \mathcal{N}_{i}(t)} \gamma_{i j}
$$

Noticing that the last term of the equation is equal to $\gamma_{i}$, we obtain the following:

$$
\dot{V}_{i} \leq-\frac{a}{\gamma_{i}} \sum_{j \in \mathcal{N}_{i}(t)} \gamma_{i j} \frac{e_{i}^{T}\left(x_{i}-x_{j}\right)}{\left\|x_{i}-x_{j}\right\|}+(a+b)\left\|e_{i}\right\| .
$$

Recalling that $\left(x_{i}-x_{j}\right)=\left(e_{i}-e_{j}\right)$, by substituting the previous equality in eq. (8), we obtain:

$$
=-\frac{a}{\gamma_{i}} \sum_{j \in \mathcal{N}_{i}(t)} \gamma_{i j} \frac{e_{i}^{T}\left(e_{i}-e_{j}\right)}{\left\|e_{i}-e_{j}\right\|}+(a+b)\left\|e_{i}\right\| .
$$

It turns out that in order to have a semi-definite negative (SDN) time derivative of the Lyapunov function, the following condition must be satisfied:

$$
-\frac{a}{\gamma_{i}} \sum_{j \in \mathcal{N}_{i}(t)} \gamma_{i j} \frac{e_{i}^{T}\left(e_{i}-e_{j}\right)}{\left\|e_{i}-e_{j}\right\|}+(a+b)\left\|e_{i}\right\| \leq 0,
$$

or, in other terms:

$$
(a+b)\left\|e_{i}\right\| \leq \frac{a}{\gamma_{i}} \sum_{j \in \mathcal{N}_{i}(t)} \gamma_{i j} \frac{e_{i}^{T}\left(e_{i}-e_{j}\right)}{\left\|e_{i}-e_{j}\right\|} .
$$

Let us now consider all the elements of the term $V_{1}$ :

$\dot{V}_{1}=\sum_{i=1}^{n}\left[-\frac{a}{\gamma_{i}} \sum_{j \in \mathcal{N}_{i}(t)} \gamma_{i j} \frac{e_{i}^{T}\left(e_{i}-e_{j}\right)}{\left\|e_{i}-e_{j}\right\|}+(a+b)\left\|e_{i}\right\|\right] \leq 0$.

The previous equation can be restated as follows:

$$
(a+b) \sum_{i=1}^{n}\left\|e_{i}\right\| \leq \sum_{i=1}^{n}\left[\frac{a}{\gamma_{i}} \sum_{j \in \mathcal{N}_{i}(t)} \gamma_{i j} \frac{e_{i}^{T}\left(e_{i}-e_{j}\right)}{\left\|e_{i}-e_{j}\right\|}\right]
$$

Basically, what we are looking for is a lower bound of the term:

$$
a \sum_{i=1}^{n}\left[w_{i} \sum_{j \in \mathcal{N}_{i}(t)} \gamma_{i j} \frac{e_{i}^{T}\left(e_{i}-e_{j}\right)}{\left\|e_{i}-e_{j}\right\|}\right],
$$

where $w_{i}=\frac{1}{\gamma_{i}}$ has been used. To this aim, let us take into account the following row-stochastic error Laplacian matrix $\hat{\mathcal{L}}$ :

$$
\hat{l}_{i j}= \begin{cases}\sum_{j \in \mathcal{N}_{i}(t)} \gamma_{i j} \frac{w_{i}}{\left\|e_{i}-e_{j}\right\|} & j=i \\ -\gamma_{i j} \frac{w_{i}}{\left\|e_{i}-e_{j}\right\|} & j \in \mathcal{N}_{i}(t) \\ 0 & \text { otherwise. }\end{cases}
$$

Equation (11) can be restated in terms of the algebraic connectivity of the Laplacian matrix $\hat{\mathcal{L}}$ as follows:

$$
a \sum_{i=1}^{n}\left[w_{i} \sum_{j \in \mathcal{N}_{i}(t)} \gamma_{i j} \frac{e_{i}^{T}\left(e_{i}-e_{j}\right)}{\left\|e_{i}-e_{j}\right\|}\right]=a e^{T} \hat{\mathcal{L}}^{d} e,
$$

where $\hat{\mathcal{L}}^{d}=\hat{\mathcal{L}} \otimes I_{d}$ and $\otimes$ denotes the Kronecker product. For the quadratic form in (13), the following inequality holds:

$a e^{T} \hat{\mathcal{L}}^{d} e \geq a \hat{\lambda}_{2}(\hat{\mathcal{L}})\|e\|^{2}, \quad \forall e \notin \operatorname{span}\left\{\mathbf{1} \otimes \xi_{1}, \ldots, \mathbf{1} \otimes \xi_{d}\right\}$

where $\xi_{i}$ is the $i$-th vector of the canonical base in $\mathbb{R}^{d}$.

It is worthy to note that $\mathbf{e} \in \operatorname{span}\left\{\mathbf{1} \otimes \xi_{1}, \ldots, \mathbf{1} \otimes \xi_{d}\right\}$ implies:

$$
\mathbf{x}=\left[x_{1}, \ldots, x_{n}\right]^{T} \in \operatorname{span}\left\{\mathbf{1} \otimes \xi_{1}, \ldots, \mathbf{1} \otimes \xi_{d}\right\},
$$

or, in other terms, $x_{1}=\ldots=x_{n}$. This case represents a trivial steady state for the interaction dynamics given in eq. (1), namely all the robots are collapsed to the same point. Furthermore, it should be pointed out that this trivial steady state could be reached only if $\mathbf{x}\left(t_{0}\right)=\left[x_{1}\left(t_{0}\right), \ldots, x_{n}\left(t_{0}\right)\right]^{T} \in \operatorname{span}\left\{\mathbf{1} \otimes \xi_{1}, \ldots, \mathbf{1} \otimes \xi_{d}\right\}$. Therefore, it turns out that $\operatorname{span}\left\{\mathbf{1} \otimes \xi_{1}, \ldots, \mathbf{1} \otimes \xi_{d}\right\}$ is an invariant subspace which is orthogonal to the swarm trajectories under the assumption that the initial conditions do not belong to such a subspace, i.e., $\mathbf{x}\left(t_{0}\right)=\left[x_{1}\left(t_{0}\right), \ldots, x_{n}\left(t_{0}\right)\right]^{T} \notin \operatorname{span}\left\{\mathbf{1} \otimes \xi_{1}, \ldots, \mathbf{1} \otimes \xi_{d}\right\}$. Let us substitute the previous equation in eq. (10). The following is obtained:

$$
(a+b) \sqrt{n}\|e\| \leq a \hat{\lambda}_{2}(\hat{\mathcal{L}})\|e\|^{2},
$$

where the fact that $\sum_{i=1}^{n}\left\|e_{i}\right\| \leq \sqrt{n}\|e\|$ has been used.

It is worth to point out that the algebraic connectivity $\hat{\lambda}_{2}(\hat{\mathcal{L}})$ is a function of the Laplacian matrix thus it depends upon the errors $e_{i}, \forall i \in V$. Nevertheless, it is possible to derive a bound which does not depend upon $e$ by recalling the Assumption 3 as follows:

$$
(a+b) \sqrt{n}\|e\| \leq a \hat{\lambda}_{2, \min }(\hat{\mathcal{L}})\|e\|^{2} .
$$

Now, since the previous inequality can be restated as:

$$
\dot{V}_{1} \leq-\|e\|\left[a \hat{\lambda}_{2, \min }(\hat{\mathcal{L}})\|e\|-(a+b) \sqrt{n}\right] \leq 0,
$$

and by recalling that $\dot{V}_{2}=0$ due to eq. (7), it follows that the derivative of the Lyapunov candidate is semi-definite negative if the following condition holds:

$$
\|e\| \geq \frac{(a+b) \sqrt{n}}{a \hat{\lambda}_{2, \min }(\hat{\mathcal{L}})}=\frac{\sqrt{n}}{\hat{\lambda}_{2, \min }(\hat{\mathcal{L}})}\left(1+\frac{b}{a}\right)
$$

Therefore, the swarm moves towards the region:

$$
\|\chi-\bar{\chi}\| \leq \frac{\sqrt{n}}{\lambda_{2, \min }(\hat{\mathcal{L}})}\left(1+\frac{b}{a}\right)
$$

with $\bar{\chi}=\mathbf{1} \otimes \bar{x}$.

Note that, the result given in Theorem 2 holds for a dynamics where each robot is assumed to have a timevarying neighborhood (eq. (1)). Therefore, no further stability analysis is required for the integration of the obstacle avoidance as it simply involves the addition/removal of a virtual robot to the neighborhood of any robot which is closer than a given threshold to an obstacle. 


\section{Time convergence}

In the following, we prove that the multi-robot system moves towards the bounded region given in Theorem 2 in finite-time.

Theorem 3: Let us consider a swarm of robots whose dynamics is described by eq. (1). Then the swarm moves arbitrarily close to the bounded region $\mathcal{B}_{r}$ defined in Theorem 2 in finite-time $t_{f}$, that is:

$$
t_{f} \leq-\frac{n}{2 \varepsilon a \hat{\lambda}_{2, \min }(\hat{\mathcal{L}})} \ln \left(\frac{\xi^{2}}{2 V(0)}\right)
$$

with $\xi$ defined as follows:

$$
\xi=(1+\eta) \frac{\sqrt{n}}{\hat{\lambda}_{2, \min }(\hat{\mathcal{L}})}\left(1+\frac{b}{a}\right)
$$

where $\eta=\frac{\varepsilon}{1-\varepsilon}$ with $\varepsilon \in(0,1)$.

Proof: In order to prove the lemma, let us consider the bound given in eq. (14):

$$
\begin{aligned}
\dot{V} \leq & -a \hat{\lambda}_{2, \min }(\hat{\mathcal{L}})\|e\|^{2}+(a+b) \sqrt{n}\|e\| \\
\leq & -\varepsilon a \hat{\lambda}_{2, \min }(\hat{\mathcal{L}})\|e\|^{2} \\
& -\|e\|\left[(1-\varepsilon) a \hat{\lambda}_{2, \min }(\hat{\mathcal{L}})\|e\|-(a+b) \sqrt{n}\right]
\end{aligned}
$$

At this point, if the following condition holds:

$$
(1-\varepsilon) a \hat{\lambda}_{2, \min }(\hat{\mathcal{L}})\|e\|-(a+b) \sqrt{n}>0
$$

that is:

$$
\|e\|>\frac{1}{(1-\varepsilon)} \frac{(a+b) \sqrt{n}}{a \hat{\lambda}_{2, \min }(\hat{\mathcal{L}})}=\frac{1}{(1-\varepsilon)} \frac{\sqrt{n}}{\hat{\lambda}_{2, \min }(\hat{\mathcal{L}})}\left(1+\frac{b}{a}\right)=\xi .
$$

Then:

$$
\begin{aligned}
\dot{V} & \leq-\epsilon a \hat{\lambda}_{2, \min }(\hat{\mathcal{L}})\|e\|^{2} \leq-\epsilon a \frac{\hat{\lambda}_{2, \min }(\hat{\mathcal{L}})}{n} \sum_{i=1}^{n}\left\|e_{i}\right\|^{2} \\
& \leq-2 \epsilon a \frac{\hat{\lambda}_{2, \min }(\hat{\mathcal{L}})}{n} V(t)
\end{aligned}
$$

Thus, the swarm moves arbitrarily close to the bounded region in finite time:

$$
t_{f} \leq-\frac{n}{2 \epsilon a \hat{\lambda}_{2, \min }(\hat{\mathcal{L}})} \ln \left(\frac{\xi^{2}}{2 V(0)}\right)
$$

\section{Algorithm Validation}

In order to validate the theoretical analysis and the effectiveness of the proposed swarm aggregation algorithm several simulations along with experiments have been carried out. The following functions and parameters are used in both the simulations and the experiments:

$$
\begin{aligned}
& \Phi(\|y\|)=\exp \left(-\frac{\|y\|^{4}}{0.02}\right), \gamma_{i}=\frac{1}{\left\|x_{i}-x_{j}\right\|^{3}}, \\
& {[-a, b]=\left[\begin{array}{ll}
-4,0.4] .
\end{array}\right.}
\end{aligned}
$$

\section{A. Simulations}

Simulations have been carried out by using the Player/Stage simulation environment, i.e., [26]. In the accompanying video, a typical example of swarm aggregation behavior for a swarm composed of 15 units moving from the left to the right within an environment filled with obstacles is shown. In the simulated environment, several obstacles are placed along with a narrow passage in order to show the ability of the swarm to move safely through the cluttered environment exhibiting a cohesive behavior.

\section{B. Experiments}

Experiments have been carried out by exploiting 5 units of the SAETTA mobile robotic platform developed at the Robotics and Sensor Fusion Lab of the Department of Computer Science and Automation at the University of "Rome Tre".

The SAETTA mobile robot is a low-cost robotic platform which features a complete sensorial system, a very accurate traction in indoor environment, and a wireless communication channel for multi-robot applications. Further details regarding the SAETTA platform can be found in [27]. Each SAETTA unit was equipped with a compass to let the swarm share a common heading reference. This allows for a human supervisor to issue guidance commands to the swarm. The supervision was limited to high-level guidance commands, e.g., "move to the north" referred to the swarm as a whole. Robots were responsible for the execution of the guidance commands while showing a cohesive behavior through local interaction. Furthermore, this central unit has been also equipped with a low-cost vision system to retrieve the relative distance among the robots. Communication between the central unit and the swarm has been realized by means of a wifi channel. Details concerning the integration of the aggregation and execution of the guidance commands and other information regarding the experimental setup can be found in [23] and references therein.

In the accompanying video, a typical example of swarm aggregation and guidance for a team composed of 5 SAETTA units in an environment filled with 4 obstacles is shown. It can be noticed by watching the video how the swarm can safely move within the environment by avoiding the obstacles while showing a cohesive behavior. Figure IV-A shows a sequence of screenshots representing four instants of the experiment we carried out. It should be noticed that obstacles were placed in such a way that only one robot could safely move within two obstacles. In particular, Figure 1(a) depicts the initial configuration for the swarm which is about to move towards the north of environment. Figure 1(b) depicts the swarm approaching an obstacle on the east of the environment, it can be noticed how the swarm is approaching the obstacle in order to move around it as a whole. Figure 1(c) depicts the swarm splitting around an obstacle. Finally, Figure 1(d) depicts the swarm moving between two obstacles. It can be noticed how the swarm, according to the fact that only one robot can safely move between two obstacles, shapes itself roughly as a line. 


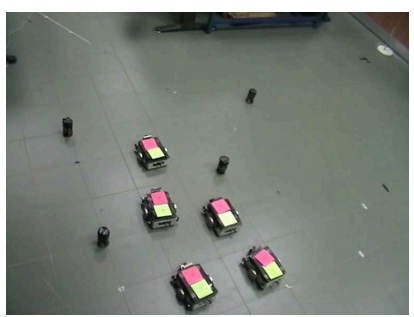

(a) Starting Configuration

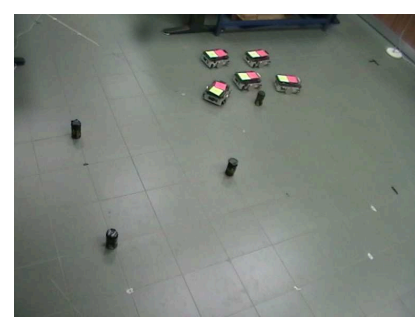

(b) Moving Around an Obstacle

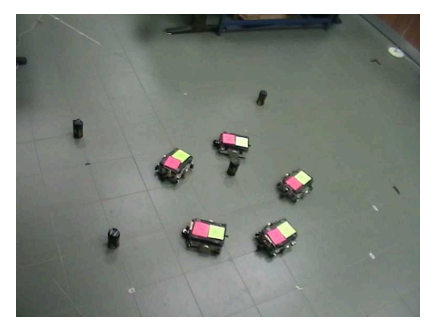

(c) Splitting Around an Obstacle

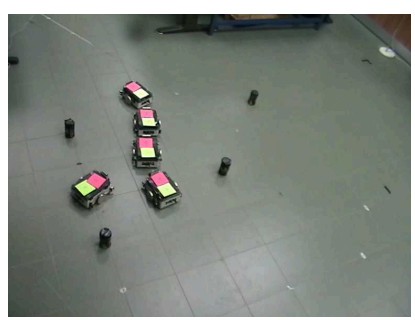

(d) Moving Between Two Obstacles

\section{CONCLUSiOnS}

In this work, we have proposed a novel decentralized swarm aggregation algorithm for multi-robot systems.

The novelties introduced in this work compared to our previous contributions [23], [24] concern the fact that the actuator saturation can be asymmetric with respect to the forward and backward velocity to better comply with the hardware/software specifications, and the fact that the obstacle avoidance algorithm can be more easily integrated within the local control law of each robot ensuring a safer navigation within environments filled with obstacles. A theoretical characterization of the properties of the proposed swarm aggregation algorithm has been provided. Furthermore, simulations along with experimental results carried out with the SAETTA mobile robotic platform developed at the Robotics and Sensor Fusion Lab of the Department of Computer Science and Automation at the University of "Rome Tre" have been provided to corroborate the theoretical results.

Future work will be focused on the integration of a connectivity maintenance algorithm within the proposed control schema and its theoretical validation.

\section{REFERENCES}

[1] P. Brass, F. Cabrera-Mora, A. Gasparri, and J. Xiao, "Multirobot tree and graph exploration," IEEE Transactions on Robotics, vol. 27, no. 4, pp. $707-717$, aug. 2011.

[2] J. Gancet, E. Motard, A. Naghsh, C. Roast, M. Arancon, and L. Marques, "User interfaces for human robot interactions with a swarm of robots in support to firefighters," in 2010 IEEE International Conference on Robotics and Automation (ICRA), may 2010.

[3] A. Gasparri, B. Krishnamachari, and G. S. Sukhatme, "A framework for multi-robot node coverage in sensor networks," Annals of Mathematics and Artificial Intelligence, vol. 52, no. 2-4, pp. 281-305, Apr. 2008. [Online]. Available: http://dx.doi.org/10.1007/ s10472-009-9126-9

[4] D. Shell and M. Mataric, "On foraging strategies for large-scale multirobot systems," in Intelligent Robots and Systems, 2006 IEEE/RSJ International Conference on, oct. 2006, pp. 2717 -2723.

[5] S. Smith, M. Schwager, and D. Rus, "Persistent robotic tasks: Monitoring and sweeping in changing environments," IEEE Transactions on Robotics, vol. 28, no. 2, pp. 410 -426, april 2012.

[6] D. Fox, W. Burgard, H. Kruppa, and S. Thrun, "A probabilistic approach to collaborative multi-robot localization," Autonomous Robots, vol. 8, pp. 325-344, 2000.

[7] S. Roumeliotis and G. Bekey, "Distributed multirobot localization," IEEE Transactions on Robotics and Automation, vol. 18, no. 5, pp. $781-795$, oct 2002.

[8] C. Parker and Z. Hong, "Consensus-based task sequencing in decentralized multiple-robot systems using local communication," in Intelligent Robots and Systems, 2008. IROS 2008. IEEE/RSJ International Conference on, sept. 2008, pp. $1421-1426$.

[9] D. D. Paola, A. Gasparri, D. Naso, G. Ulivi, and F. Lewis, "Decentralized task sequencing and multiple mission control for heterogeneous robotic networks," in Robotics and Automation (ICRA), 2011 IEEE International Conference on, may 2011, pp. 4467 -4473.

[10] M. Egerstedt and H. Xiaoming, "Formation constrained multi-agent control," IEEE Transactions on Robotics and Automation,, vol. 17, no. 6, pp. 947 - 951, dec 2001.

[11] D. Wenjie and J. Farrell, "Cooperative control of multiple nonholonomic mobile agents," IEEE Transactions on Automatic Control, vol. 53, no. 6, pp. $1434-1448$, july 2008 .

[12] Y. Peng, R. Freeman, and K. Lynch, "Multi-agent coordination by decentralized estimation and control," IEEE Transactions on Automatic Control, vol. 53, no. 11, pp. $2480-2496$, dec. 2008.

[13] J. Qin, W. X. Zheng, and H. Gao, "Coordination of multiple agents with double-integrator dynamics under generalized interaction topologies," IEEE Transactions on Systems, Man, and Cybernetics, Part B: Cybernetics, , vol. 42, no. 1, pp. 44 -57, feb. 2012.

[14] S. Erol and W. Alan, "Special issue on swarm robotics," Swarm Intelligence, vol. 2, no. 2-4, pp. 69-72, 2008.

[15] V. Gazi and K. Passino, Swarm Stability and Optimization. Springer, 2011.

[16] — "Stability analysis of swarms," IEEE Transactions on Automatic Control, vol. 48, no. 4, pp. 692 - 697, april 2003.

[17] V. Gazi, "Swarm aggregations using artificial potentials and slidingmode control," IEEE Transactions on Robotics, vol. 21, no. 6, pp. $1208-1214$, dec. 2005.

[18] D. Dimarogonas and K. Kyriakopoulos, "Connectedness preserving distributed swarm aggregation for multiple kinematic robots," IEEE Transactions on Robotics, vol. 24, no. 5, pp. 1213 -1223, oct. 2008.

[19] S. Das, U. Halder, and D. Maity, "Chaotic dynamics in social foraging swarms - an analysis," IEEE Transactions on Systems, Man, and Cybernetics, Part B: Cybernetics, vol. 42, no. 4, pp. $1288-1293$, aug. 2012.

[20] J. Lawton, R. Beard, and B. Young, "A decentralized approach to formation maneuvers," IEEE Transactions on Robotics and Automation, vol. 19, no. 6, pp. 933 - 941, dec. 2003.

[21] L. Bo and Y. Hai, "Flocking in multi-agent systems with a bounded control input," in International Workshop on Chaos-Fractals Theories and Applications. IWCFTA '09., nov. 2009, pp. $130-134$.

[22] D. Kostic, S. Adinandra, J. Caarls, N. van de Wouw, and H. Nijmeijer, "Saturated control of time-varying formations and trajectory tracking for unicycle multi-agent systems," in 2010 49th IEEE Conference on Decision and Control (CDC), dec. 2010, pp. $4054-4059$.

[23] A. Gasparri, A. Priolo, and G. Ulivi, "A swarm aggregation algorithm for multi-robot systems based on local interaction," in Proceedings of the IEEE Multi-Conference on Systems and Control, 2012.

[24] A. Gasparri, A. Priolo, G. Oriolo, and G. Ulivi, "A swarm aggregation algorithm for multi-robot systems with actuator saturation based on local interaction," in IEEE/RSJ International Conference on Intelligent Robots and Systems, 2012.

[25] J.-C. Latombe, Robot Motion Planning. Norwell, MA, USA: Kluwer Academic Publishers, 1991.

[26] R. Vaughan, "Massively multi-robot simulation in stage," Swarm Intelligence, vol. 2, no. 2-4, pp. 189-208, 2008.

[27] M. D. Rocco, F. L. Gala, and G. Ulivi, "Seatta: A small and cheap mobile unit to test multirobot algorithms," Robotics Automation Magazine, IEEE, vol. PP, no. 99, p. 1, 2012. 